\title{
Características da sucção não-nutritiva em RN a termo e pré-termo tardio
}

\author{
Characteristics of non-nutritive sucking in full-term and late \\ preterm infants
}

\author{
Ana Paula d'Oliveira Gheti Kao', Zelita Caldeira Ferreira Guedes², Amélia Miyashiro Nunes dos Santos ${ }^{3}$
}

\begin{abstract}
RESUMO
Objetivo: Comparar os parâmetros de sucção não nutritiva de recém-nascidos a termo e pré-termo tardio. Métodos: Os recémnascidos foram divididos em dois grupos, pré-termo tardio (RNPT tardio) e a termo (RN a termo) e, submetidos à avaliação da sucção não-nutritiva utilizando-se um protocolo adaptado da Escala de Avaliação Motora Oral. Foi realizada análise estatística para comparação dos grupos. Resultados: Os reflexos de procura e de sucção foram menos frequentes nos RNPT tardio, comparados aos RN a termo, assim como a preensão palmar e mãos em linha média. A maioria dos RNPT tardio apresentou sono leve ou estava sonolento antes da avaliação. Os RNPT tardio apresentaram predominantemente sucção esporádica ou grupos de sucção com pausas longas e travamento e/ou tremores de mandíbula. A retração de língua e a protrusão de língua foram mais presentes nos RNPT tardio e o canolamento de língua nos RN a termo. Conclusão: Prontidão para a mamada, estado comportamental, postura corporal, padrão e força de sucção e movimentos de língua foram os parâmetros menos frequentes nos RNPT tardio em relação aos RN a termo.
\end{abstract}

Descritores: Recém-nascido; Prematuro; Peso ao nascer; Comportamento de sucção; Idade gestacional; Aleitamento materno

\section{INTRODUÇÃO}

Recém-nascido pré-termo tardio (RNPT tardio) é definido como prematuro de 34 semanas a 36 semanas e 6 dias de gestação contadas a partir do primeiro dia do último período menstrual normal da mãe ${ }^{(1)}$. O número de RNPT tardio tem aumentado nos últimos anos devido às técnicas obstétricas de fertilização assistida. Em 2005, nos Estados Unidos, 70\% dos prematuros eram $\operatorname{tardio}^{(2)}$. Em 2004, um estudo em Pelotas encontrou $10,8 \%$ de RNPT tardio nesta região $0^{(3)}$. Por terem o peso e o tamanho de alguns recém-nascidos a termo, o prematuro tardio é considerado por muitos profissionais, como neonato com o desenvolvimento normal e baixa morbidade. No entanto no período neonatal pode apresentar imaturidade,

Trabalho realizado no Departamento de Fonoaudiologia e Disciplina de Pediatria Neonatal, Universidade Federal de São Paulo - UNIFESP - São Paulo (SP), Brasil.

(1) Programa de Pós-Graduação (Mestrado) em Distúrbios da Comunicação Humana, Departamento de Fonoaudiologia, Universidade Federal de São Paulo - UNIFESP - São Paulo (SP), Brasil.

(2) Departamento de Fonoaudiologia, Universidade Federal de São Paulo UNIFESP - São Paulo (SP), Brasil.

(3) Departamento de Pediatria, Universidade Federal de São Paulo - UNIFESP - São Paulo (SP), Brasil.

Endereço para correspondência: Ana Paula d'Oliveira Gheti Kao. R. Botucatu, 802, Vila Mariana, São Paulo (SP), Brasil, CEP: 04023-062. Email: ana.kao@ig.com.br

Recebido em: 1/7/2010; Aceito em: 17/8/2010 alterações respiratórias, instabilidade de temperatura, kecnicterus, convulsões, hipoglicemia, hiperbilirrubinemia, falta de controle dos mecanismos do sono, dificuldades alimentares e taxas mais elevadas de re-internação ${ }^{(1,4)}$.

A função oromotora ocupa, na maioria das vezes, um papel secundário na escala de prioridades dentro da reabilitação e prevenção de alterações no recém-nascido. No entanto, é prérequisito para o desenvolvimento físico e neuropsicomotor do neonato, uma vez que se relaciona à ingestão de nutrientes, interação com o meio e até mesmo com o desenvolvimento da fala ${ }^{(5)}$.

$\mathrm{Na}$ área fonoaudiológica, protocolos têm sido utilizados com o objetivo de avaliar a sucção e contribuir para o planejamento da intervenção terapêutica em caso de dificuldades alimentares ${ }^{(6-8)}$. No entanto, os estudos se concentram nos prematuros com idade gestacional inferior a 34 semanas $^{(9-11)}$.

Neste contexto, o presente estudo tem como objetivo comparar os parâmetros de sucção não-nutritiva de $\mathrm{RN}$ a termo e RNPT tardio.

\section{MÉTODOS}

Estudo transversal com neonatos de 24 a 72 horas de vida nascidos em dois hospitais públicos universitários de São Paulo (SP), no período de setembro de 2007 a março de 2009.

A pesquisa foi aprovada pelo Comitê de Ética em Pesquisa da Universidade Federal de São Paulo (CEP 0928/06) e os pais 
ou responsável legal dos recém-nascidos assinaram o Termo de Consentimento.

\section{Critérios de inclusão}

Foram incluídos recém-nascidos com 24 a 72 horas de vida, com idade gestacional de 34 a 41 semanas e seis dias, independente do peso de nascimento, estáveis clinicamente, sem necessidade de oxigenoterapia e com prescrição de alimentação por via oral por mamadeira ou seio materno.

\section{Critérios de exclusão}

Foram excluídos recém-nascidos cujas mães apresentaram hipertensão arterial crônica, pré-eclâmpsia, amniorrexe maior que 18 horas antes do parto, presença de líquido amniótico meconial, sinais e/ou sintomas de corioamnionite, recém-nascidos com anomalias congênitas, malformações craniofaciais, sinais/ sintomas de infecção, doenças respiratórias, cardiovasculares, gastrintestinais ou neurológicas ou que necessitassem de cuidados em unidades de médio ou alto risco ${ }^{(10,12)}$.

\section{Procedimentos}

Para inclusão dos recém-nascidos no estudo, a pesquisadora comparecia duas vezes por semana na unidade neonatal e selecionava os neonatos que obedeciam aos critérios de inclusão. Para cada prematuro incluído, selecionava dois recém-nascidos a termo entre aqueles cujo número de registro hospitalar era ímpar.

Os recém-nascidos que obedeceram aos critérios de inclusão foram divididos em dois grupos: recém-nascidos com idade gestacional de 34 semanas a 36 semanas e 6 dias (RNPT tardio); recém-nascidos a termo com idade gestacional de 37 semanas a 41 semanas e 6 dias ( $\mathrm{RN}$ a termo).

No período do estudo, nasceram 124 RNPT tardio e 318 RN a termo. Destes, foram incluídos 40 (32,3\%) RNPT tardio e 85 $(26,7 \%)$ a termo. Dessa forma, foram estudados 125 neonatos.

Dos recém-nascidos estudados, $85(68,0 \%)$ eram a termo e $40(32,0 \%)$ RNPT tardio, sendo $62(49,6 \%)$ do gênero masculino.

A idade gestacional dos RNPT tardio variou de 34 semanas a 36 semanas e 6 dias e peso ao nascer de 1385 a 3380 gramas. Já os RN a termo apresentaram idade gestacional de 37 semanas a 41 semanas e 1 dia e peso ao nascer de 1765 a 4100 gramas.

Os recém-nascidos incluídos no estudo foram submetidos à avaliação da sucção não-nutritiva (SNN), utilizando-se um protocolo adaptado da Escala de Avaliação Motora Oral ${ }^{(6)}$ (Quadro 1). O recém-nascido foi avaliado dentro da incubadora ou no berço comum, com a cabeça apoiada na mão direita da pesquisadora e mantendo leve flexão corporal, 15 minutos antes do horário regular da alimentação das 15:00 horas. Além disso, foram coletados dados demográficos e clínicos das mães e dos recém-nascidos.

\section{Análise estatística}

As variáveis categóricas foram expressas em número e frequência e comparadas por Qui-quadrado $\left(\chi^{2}\right)$ ou teste exato de Fisher. Para análise dos fatores associados à alteração da SNN realizou-se a regressão logística univariada, na qual se considerou a variável RNPT tardio como independente e os parâmetros relativos à SNN como variável resposta. Considerou-se $\mathrm{p}<0,05$ como nível de significância estatística.

\section{RESULTADOS}

As características demográficas e clínicas dos recémnascidos incluídos no estudo estão expostas na Tabela 1.

Tabela 1. Características demográficas e clínicas dos recém-nascidos incluídos no estudo

\begin{tabular}{|c|c|c|c|}
\hline & $\begin{array}{l}\text { RNPT tardio } \\
\quad(n=40)\end{array}$ & $\begin{array}{l}\text { RN a termo } \\
\quad(n=85)\end{array}$ & Valor de $p$ \\
\hline Gênero masculino $n(\%)^{a}$ & $17(42,5)$ & $45(52,9)$ & 0,276 \\
\hline IG (semanas) ${ }^{b}$ & $35,7 \pm 0,9$ & $39,0 \pm 1,1$ & $<0,001^{\star}$ \\
\hline Peso $(g)^{b}$ & $2507 \pm 437$ & $3112 \pm 455$ & $<0,001^{*}$ \\
\hline RN PIG n (\%) & $12(30,0)$ & $5(6,3)$ & 0,055 \\
\hline Apgar $1^{\circ}$ minuto $^{b}$ & $8,3 \pm 0,8$ & $8,3 \pm 0,8$ & 0,763 \\
\hline Apgar $5^{\circ}$ minuto $^{\mathrm{b}}$ & $9,3 \pm 0,6$ & $9,3 \pm 0,6$ & 0,883 \\
\hline Parto cesáreo n (\%)a & $16(40,0)$ & $39(45,9)$ & 0,537 \\
\hline
\end{tabular}

*Valores significativos $(p \leq 0,05)$

a Teste Qui quadrado

${ }^{b}$ Teste $t$

Legenda: RNPT = recém-nascido pré-termo; RN = recém-nascido; $I G$ = idade gestacional; RN PIG = recém-nascido pequeno para a idade gestacional

A presença de parâmetros de SNN entre o RN a termo e RNPT tardio e a razão de chance do RNPT tardio apresentar tal parâmetro, comparado ao RN a termo estão expostas na Tabela 2. Nessa avaliação, os neonatos poderiam apresentar mais de um parâmetro.

Em relação ao tônus corporal, 38 (95,0\%) RNPT tardio apresentaram tônus adequado. $\mathrm{O}$ mesmo foi observado em 85 $(100 \%)$ nascidos a termo, sem diferença estatística $(p=0,060)$.

Quanto ao estado comportamental do recém-nascido no início da avaliação, a maioria dos RNPT tardio apresentou sono leve ou estava sonolento antes da avaliação. No entanto, o RN a termo apresentou predominantemente estado de alerta. A chance do RNPT tardio estar alerta foi 5,71(IC95\%: 2,35213,888 ) vezes menor que a do $\mathrm{RN}$ a termo.

A postura corporal exibida pelos neonatos foi verificada no início da avaliação. Cada neonato poderia exibir mais do que um padrão postural. O RNPT tardio apresentou preensão palmar e mãos em linha média com menor frequência que o RN a termo. A chance do RNPT tardio apresentar preensão palmar foi 2,65 (IC95\%: 1,221-5,708) vezes menor que o $\mathrm{RN}$ a termo e a sua chance de apresentar mãos em linha média foi 3,69 (IC95\%: 1,552-8,771) vezes menor, comparados aos a termo.

Quanto ao padrão de sucção dos recém-nascidos, o RNPT tardio apresentou predominantemente sucção esporádica ou grupos de sucção com pausas longas e travamento e/ou tremores de mandíbula. Já o RN a termo apresentou grupos de sucção acompanhados de pausas. Nenhum neonato dos dois grupos apresentou excursão exagerada de mandíbula. 
Quadro 1. Parâmetros utilizados para avaliação da sucção não-nutritiva

\begin{tabular}{|c|c|c|}
\hline Parâmetros & Descrição & \\
\hline Prontidão para mamada & $\begin{array}{l}\text { Choro } \\
\text { Reflexo de procura } \\
\text { Reflexo de sucção }\end{array}$ & $\begin{array}{l}\text { Toque com o dedo mínimo enluvado na região peri-oral, considerando-se } \\
\text { presente quando o RN virou a cabeça em direção ao estímulo. } \\
\text { Toque com o dedo mínimo da avaliadora na língua, considerando-se presente } \\
\text { se o RN iniciou a sucção. }\end{array}$ \\
\hline Tônus corporal & $\begin{array}{l}\text { Adequado } \\
\text { Hipotônico } \\
\text { Hipertônico }\end{array}$ & \\
\hline Estado de consciência ${ }^{(13)}$ & $\begin{array}{l}\text { Alerta } \\
\text { Sono leve } \\
\text { Sonolento } \\
\text { Sono profundo }\end{array}$ & $\begin{array}{l}\text { Acordado com movimentos mínimos ou vigorosos. } \\
\text { Olhos fechados, algum movimento corporal. } \\
\text { Olhos abrindo e fechando. } \\
\text { Sem movimentos, respiração regular. }\end{array}$ \\
\hline $\begin{array}{l}\text { Postura no início da } \\
\text { avaliação }\end{array}$ & $\begin{array}{l}\text { Estável com apoio } \\
\text { Desorganizado } \\
\text { Mãos a face } \\
\text { Preensão palmar } \\
\text { Mãos em linha média }\end{array}$ & $\begin{array}{l}\text { Membros superiores e inferiores voltados à linha média; cabeça e pescoço } \\
\text { apoiados na mão da examinadora. } \\
\text { Presença de membros superiores e inferiores desorganizados, em extensão, } \\
\text { fora do eixo médio corporal. } \\
\text { Mãos voltadas à face, podendo tocá-las. } \\
\text { Após o toque da examinadora na palma da mão do RN, este fecha a mão. } \\
\text { Mãos centralizadas no eixo central do corpo }\end{array}$ \\
\hline Sucção & $\begin{array}{l}\text { Sucção esporádica } \\
\text { Grupos de sucção } \\
\text { Pausas } \\
\text { Pausas longas } \\
\text { Ajudar a dar pausas } \\
\text { Travamento/tremores } \\
\text { Excursão exagerada de mandíbula }\end{array}$ & $\begin{array}{l}\text { Poucas sucções, sem ritmo. } \\
\text { Presença de sucções intercaladas com pausas e apresentando ritmo. } \\
\text { Durando até } 10 \text { segundos. } \\
\text { Durando mais que } 10 \text { segundos. } \\
\text { Precisou de ajuda da examinadora para dar pausas e não se desorganizar. } \\
\text { Presença de tremores ou travamento de mandíbula após um período de sucções. } \\
\text { Rebaixamento de mandíbula exagerado. }\end{array}$ \\
\hline Força da sucção & Forte ou não & \\
\hline Movimentos de língua & $\begin{array}{l}\text { Protrusão/retração } \\
\text { Tremores } \\
\text { Canolamento }\end{array}$ & $\begin{array}{l}\text { Presença de movimento ântero-posterior de língua ao sugar } \\
\text { Presença de sulco central na língua ao sugar. }\end{array}$ \\
\hline Sinais de estresse & $\begin{array}{l}\text { Alteração respiratória } \\
\text { Cianose } \\
\text { Bater asa do nariz } \\
\text { Adormecer }\end{array}$ & $\begin{array}{l}\text { Alteração do ritmo respiratório. } \\
\text { Mudança da coloração da pele e da região peri-oral. } \\
\text { Movimentação da asa do nariz. }\end{array}$ \\
\hline
\end{tabular}

Fonte: Adaptado de: Xavier C. Avaliação da alimentação de RN em fase de hospitalização (escala de avaliação motora oral da Irmandade Santa Casa de Misericórdia de São Paulo). Pró-Fono. 1995;7(2):69-74.

A chance do RNPT tardio apresentar sucção esporádica, pausas longas, e travamento de língua e tremores foi respectivamente, 7,44 (IC95\%: 3,063-18,047), 4,59 (IC95\%: 1,971-10,679) e 4,15 (IC95\%: 1,780-9,674) vezes maior, quando comparada ao RN a termo. Já a chance de apresentar grupos de sucção e pausas adequadas foi, respectivamente, 8,20 (IC95\%: 3,378-20,000) e 5,29 (IC95\%: 2,336-12,048) vezes menor que o $\mathrm{RN}$ a termo.

Em relação à intensidade da força de sucção, 20 (50,0\%)
RN PT tardio e $70(82,4 \%)$ apresentaram sucção forte (Quiquadrado, $\mathrm{p}<0,001)$. A chance do RNPT tardio apresentar sucção forte foi 4,67 (IC95\%: 2,03-10,75) vezes menor que no RN a termo.

Quanto às características do movimento de língua durante a sucção, a retração e a protrusão de língua foram mais presentes no RNPT tardio e o canolamento de língua no RN a termo. No RNPT tardio, a chance de apresentar retração de língua foi 3,65 (IC95\%: 1,335-9,982) vezes maior e a chance de apresentar 
Tabela 2. Presença dos parâmetros de sucção não-nutritiva nos recém-nascidos

\begin{tabular}{|c|c|c|c|c|}
\hline & $\begin{array}{c}\text { RNPT tardio }(\mathrm{n}=40) \\
\mathrm{n}(\%)\end{array}$ & $\begin{array}{c}\text { RN termo }(n=85) \\
n(\%)\end{array}$ & OR (IC 95\%) & Valor de $p$ \\
\hline Choro $^{\mathrm{a}}$ & $7(17,5)$ & $21(24,7)$ & $0,646(0,249-1,677)$ & 0,367 \\
\hline $\mathrm{RP}^{\mathrm{a}}$ & $17(42,5)$ & $58(68,2)$ & $0,344(0,158-0,747)$ & $0,006^{*}$ \\
\hline $\mathrm{RS}^{\mathrm{a}}$ & $28(70,0)$ & $78(92,9)$ & $0,179(0,062-0,524)$ & $0,001^{*}$ \\
\hline Alerta $^{a}$ & $8(20,0)$ & $50(58,8)$ & $0,175(0,072-0,425)$ & $<0,001^{\star}$ \\
\hline Sono leve ${ }^{a}$ & $16(40,0)$ & $24(28,2)$ & $1,694(0,769-3,731)$ & 0,188 \\
\hline Sonolento $^{\mathrm{a}}$ & $16(40,0)$ & $11(12,9)$ & $4,485(1,832-10,978)$ & $0,001^{*}$ \\
\hline Estável com apoio ${ }^{a}$ & $38(95,0)$ & $83(97,6)$ & $0,458(0,062-3,374)$ & 0,592 \\
\hline Desorganizado ${ }^{b}$ & $2(5,0)$ & $2(2,4)$ & $2,184(0,296-16,094)$ & 0,592 \\
\hline Mãos a face ${ }^{a}$ & $9(22,5)$ & $24(28,2)$ & $0,738(0,306-1,778)$ & 0,497 \\
\hline Preensão palmar ${ }^{a}$ & $19(47,5)$ & $60(70,6)$ & $0,377(0,173-0,819)$ & $0,013^{*}$ \\
\hline Mãos em LMa & $24(60,0)$ & $72(84,7)$ & $0,271(0,114-0,644)$ & $0,002^{*}$ \\
\hline Sucção esporádicaa & $21(52,5)$ & $11(12,9)$ & $7,435(3,063-18,047)$ & $<0,001^{\star}$ \\
\hline Grupos de sucção ${ }^{a}$ & $18(45,0)$ & $74(87,1)$ & $0,122(0,050-0,296)$ & $<0,001^{\star}$ \\
\hline Pausas $^{a}$ & $12(30,0)$ & $59(69,4)$ & $0,189(0,083-0,428)$ & $<0,001^{\star}$ \\
\hline Pausas longas $^{a}$ & $19(47,5)$ & $14(16,5)$ & $4,588(1,971-10,679)$ & $<0,001^{\star}$ \\
\hline Ajudar a dar pausas ${ }^{* * b}$ & $1(2,5)$ & $4(4,7)$ & $0,519(0,056-4,802)$ & 1,000 \\
\hline Travamento/tremores ${ }^{\star \star \star a ~}$ & $18(45,0)$ & $14(16,5)$ & $4,149(1,780-9,674)$ & $<0,001^{\star}$ \\
\hline Protrusão & $10(25,0)$ & $8(9,4)$ & $3,208(1,156-8,905)$ & $0,021^{*}$ \\
\hline Retração $^{a}$ & $11(27,5)$ & $8(9,4)$ & $3,651(1,335-9,982)$ & $0,009^{*}$ \\
\hline Tremores $^{b}$ & $5(12,5)$ & $3(3,5)$ & $3,905(0,884-17,240)$ & 0,109 \\
\hline Canolamento $^{\mathrm{a}}$ & $28(70,0)$ & $76(89,4)$ & $0,276(0,105-0,727)$ & $0,007^{\star}$ \\
\hline Alt. Respiratóriab & $1(1,50)$ & $1(1,12)$ & $2,154(0,131-5,338)$ & 0,539 \\
\hline Adormecer ${ }^{\mathrm{a}}$ & $26(65,5)$ & $44(51,8)$ & $1,731(0,796-3,762)$ & 0,164 \\
\hline
\end{tabular}

* Valores significativos $(p \leq 0,05)$

a Teste Qui-quadrado

b Teste exato de Fisher

Legenda: $\mathrm{RNPT}$ = recém-nascido pré-termo; $\mathrm{RN}$ = recém-nascido; $\mathrm{OR}=$ razão de chances; IC = intervalo de confiança; $\mathrm{RP}=$ reflexo de procura; $\mathrm{RS}=$ reflexo de sucção; LM = linha média; ** é preciso ajudar a dar pausas para o RN não se desorganizar; ${ }^{\star \star \star}$ referentes à mandíbula

canolamento de língua foi 3,62 (IC95\%: 1,375 - 9,523) vezes menor, quando comparado ao RN a termo.

Ao final da avaliação, foram registrados os sinais de estresse, não havendo diferenças significantes entre os grupos em relação às variáveis alterações respiratórias e adormecer. Nenhum recém-nascido estudado apresentou batimento de asa de nariz ou cianose.

\section{DISCUSSÃO}

No recém-nascido, o comportamento motor oral adequado é um pré-requisito para o desenvolvimento neuropsicomotor e físico, uma vez que está relacionado à alimentação e a interação com o meio ${ }^{(5,8)}$. O desenvolvimento da sucção nãonutritiva possibilita ao recém-nascido uma sucção nutritiva adequada. A sucção não-nutritiva em prematuros estimula o batimento cardíaco ${ }^{(14,15)}$, propicia adequação da musculatura oral, regulação dos estados de consciência, facilita a digestão, transição para alimentação por via oral mais rápida e fácil, alta hospitalar precoce e maior ganho de peso ${ }^{(16)}$.

$\mathrm{Na}$ avaliação fonoaudiológica por estimulação da sucção não-nutritiva deve-se considerar os seguintes aspectos: estado de alerta, prontidão, padrão de organização corporal, aceitação de estímulo, reflexo de procura e sucção, observação de sinais de estresse como alteração do padrão respiratório, tremores de língua e mandíbula, redução do número de sucção com pausas mais prolongadas entre outros e incoordenação sucção/ deglutição/respiração ${ }^{(17)}$. Tais parâmetros foram utilizados na avaliação do presente estudo ${ }^{(6)}$.

Houve diferença significativa para reflexo de procura e de sucção entre os dois grupos estudados, com menor frequência no RNPT tardio em relação ao RN a termo. Os reflexos orais têm o papel de regulação, acalmamento e desenvolvimento motor-oral para uma sucção efetiva e podem não estar maduros no RNPT ${ }^{(17,18)}$. O reflexo de procura apresenta-se bem desenvolvido a partir de 32 semanas de idade gestacional e o reflexo de sucção a partir de 34 semanas, justificando o que foi encontrado no presente estudo ${ }^{(8,19)}$.

Em relação ao tônus corporal, não houve diferença estatística entre os dois grupos. A alteração no tônus pode levar a uma sucção fraca ou descoordenada ${ }^{(6,20)}$.

$\mathrm{O}$ estado de consciência do recém-nascido influencia no desempenho motor oral ${ }^{(6,11,20)}$. No presente estudo, o RNPT tardio apresentou sono leve ou estavam sonolentos antes da 
avaliação, similar a outro estudo ${ }^{(11)}$. No entanto, o RN a termo apresentou-se predominantemente em estado de alerta, havendo diferença estatística entre os dois grupos.

Quanto à postura corporal do recém-nascido, a chance do RNPT tardio apresentar preensão palmar e mãos em linha média foi menor quando esse grupo foi comparado ao $\mathrm{RN}$ a termo. A postura flexora favorece a organização corporal importante para sucção, possibilitando o selamento labial e movimentos adequados do complexo orofacial no momento da alimentação, além de evitar situações que comprometem o desempenho oral e que podem colocar a vida do recémnascido em risco ${ }^{(21)}$.

Em relação ao padrão de sucção do recém-nascido, o RNPT tardio apresentou predominantemente sucção esporádica ou grupos de sucção com pausas longas e travamento e/ou tremores de mandíbula. No RN a termo observou-se sequências de sucção acompanhadas de pausas, com diferença entre os dois grupos.

Em relação à intensidade da força de sucção, a chance do RNPT tardio apresentar sucção forte foi menor em relação ao $\mathrm{RN}$ a termo. A imaturidade neurológica e a falta de resposta a estímulos sensoriais presentes no prematuro justificam a presença de sucção fraca ${ }^{(22)}$.

Durante a sucção, o RNPT tardio apresenta maior chance de apresentar retração e canolamento de língua quando comparados ao RN a termo. A presença do canolamento de língua é importante para uma boa sucção ${ }^{(17,18)}$.

Ao final da avaliação, foram registrados os sinais de estresse observados nos neonatos. Não houve diferença significante entre o RNPT tardio e RN a termo em relação às variáveis alterações respiratórias e adormecer.

Algumas limitações desse estudo devem ser mencionadas. $\mathrm{O}$ fato de a pesquisadora estar presente somente duas vezes por semana e apenas no horário da mamada das $15 \mathrm{hs}$ dificultou a coleta de dados. Muitos recém-nascidos que apresentavam critérios de inclusão no estudo receberam alta hospitalar nos dias de ausência da pesquisadora. Além disso, algumas vezes os neonatos já haviam mamado ou estavam dormindo no momento da avaliação, impedindo a sua realização. Apesar disso, esse é o primeiro estudo que avalia o RNPT tardio em relação à sucção não-nutritiva mostrando uma diferença significante em relação ao RN a termo.

\section{CONCLUSÃO}

O RNPT tardio apresentou parâmetros de sucção nãonutritiva diferentes do RN a termo. Essa informação poderá contribuir para melhorar a assistência a esses recém-nascidos, principalmente do ponto de vista do fonoaudiólogo, propiciando melhor desenvolvimento global e facilitando a alta hospitalar precoce. Mais estudos serão necessários nessa linha, com maior tamanho de amostra.

\section{AGRADECIMENTOS}

À Dra Ana Lúcia Acquesta, coordenadora da Unidade Neonatal do Hospital Geral de Pirajussara, e à equipe neonatal do Hospital São Paulo pelo apoio durante a realização desse estudo.

\begin{abstract}
Purpose: To compare non-nutritive sucking parameters between late preterm and full-term infants. Methods: Infants were divided into two groups, full-term and late preterm, and were submitted to non-nutritive sucking assessment using a protocol adapted from the Oral Motor Assessment Scale. Statistical analysis was conducted for comparison between the groups. Results: The seeking and sucking reflexes were less frequent in late preterm than in full-term newborns, as well as palmar grip and hands in the midline. Most late preterm infants presented light sleep or drowsiness before the assessment. Late preterm subjects predominantly presented sporadic sucking or blocks of sucking with long pauses and mandibular locking and/or tremors. Tongue retraction and protrusion were mostly present in late preterm infants, and tongue central groove formation, in full-term infants. Conclusion: Readiness for feeding, behavioral state, axial tonus, sucking pattern and strength, and tongue movements were the less frequent parameters in late preterm infants, in comparison to full-term infants.
\end{abstract}

Keywords: Infant, newborn; Infant, premature; Sucking behavior; Gestational age; Birth weight; Breastfeeding

\title{
REFERÊNCIAS
}

1. Engle WA. A recommendation for the definition of "late preterm" (nearterm) and the birth weight-gestational age classification system. Semin Perinatol. 2006;30(1):2-7.

2. Engle WA, Tomashek KM, Wallman C; Committee on Fetus and Newborn, American Academy of Pediatrics. "Late-preterm" infants: a population at risk. Pediatrics. 2007;120(6):1390-401.

3. Santos IS, Matijasevich A, Silveira MF, Sclowitz IK, Barros AJ, Victora CG, Barros FC. Associated factors and consequences of late preterm births: results from the 2004 Pelotas birth cohort. Paediatr Perinat Epidemiol. 2008;22(4):350-9.
4. Darcy AE. Complications of the late preterm infant. J Perinat Neonatal Nurs. 2009;23(1):78-86

5. Neiva FC. Proposta de um formulário de avaliação da sucção de RN. Pró-Fono. 2000;12(2):113-9.

6. Xavier C. Avaliação da alimentação de RN em fase de hospitalização (escala de avaliação motora oral da Irmandade Santa Casa de Misericórdia de São Paulo). Pró-Fono. 1995;7(2):69-74.

7. Law-Morstatt L, Judd DM, Snyder P, Baier RJ, Dhanireddy R. Pacing as a treatment technique for transitional sucking patterns. J Perinatol. 2003;23(6):483-8. 
8. Neiva FC, Leone CR. Development of sucking rhythm and the influence of stimulation in premature infants. Pró-Fono. 2007;19(3):241-8.

9. Medoff-Cooper B, McGrath JM, Bilker W. Nutritive sucking and neurobehavioral development in preterm infants from 34 weeks PCA to term. MCN Am J Matern Child Nurs. 2000;25(2):64-70.

10. Fucile S, Gisel EG, Lau C. Effect of an oral stimulation program on sucking skill maturation of preterm infants. Dev Med Child Neurol. 2005;47(3):158-62.

11. Fujinaga CI, Zamberlan NE, Rodarte MD, Scochi CG. Reliability of an instrument to assess the readiness of preterm infants for oral feeding. Pró-Fono. 2007;19(2):143-50.

12. Medoff-Cooper B, McGrath JM, Shults J. Feeding patterns of full-term and preterm infants at forty weeks postconceptional age. J Dev Behav Pediatr. 2002;23(4):231-6.

13. Brazelton TB. [Neonatal behavior evaluation scale]. Neuropsychiatr Enfance Adolesc. 1983;31(2-3):61-96.

14. Cohen M, Brown DR, Myers MM. Cardiovascular responses to pacifier experience and feeding in newborn infants. Dev Psychobiol. 2001;39(1):34-9.

15. Morren G, Van Huffel S, Helon I, Naulaers G, Daniëls H, Devlieger H, Casaer P. Effects of non-nutritive sucking on heart rate, respiration and oxygenation: a model-based signal processing approach. Comp Biochem Physiol A Mol Integr Physiol. 2002;132(1):97-106.

16. Caetano LC, Fujinaga CI, Scochi CG. Sucção não-nutritiva em bebês prematuros: estudo bibliográfico. Rev Lat Am Enferm. 2003;11(2):232-6.
17. Rios IJ, Oliveira MB, Farias PT, Barcellos SF, Tini V. Amamentando o Prematuro. In: Hitos SF, Periotto MC. Amamentação - Atuação fonoaudiológica: uma abordagem prática e atual. Rio de Janeiro: Revinter; 2009.p.101-7.

18. Periotto MC. Amamentação e desenvolvimento do sistema estomatognático. In: Hitos SF, Periotto MC. Amamentação - Atuação fonoaudiológica: uma abordagem prática e atual. Rio de Janeiro: Revinter; 2009. p.37-9.

19. Lau C, Alagugurusamy R, Schanler RJ, Smith EO, Shulman RJ. Characterization of the developmental stages of sucking in preterm infants during bottle feeding. Acta Paediatr. 2000;89(7):846-52.

20. Delgado SE, Halpern R. Amamentação de prematuros com menos de 1500 gramas: funcionamento motor-oral e apego. Pró-Fono. 2005;17(2):141-52.

21. Telles MS. Importância do posicionamento do bebê no momento da alimentação. In: Hitos SF, Periotto MC. Amamentação - Atuação fonoaudiológica: uma abordagem prática e atual. Rio de Janeiro: Revinter; 2009. p.87-96.

22. Meier PP, Furman LM, Degenhardt M. Increased lactation risk for late preterm infants and mothers: evidence and management strategies to protect breastfeeding. J Midwifery Womens Health. 2007;52(6):579-87. 